\title{
Which Place and Roles of the History of Science in Moroccan Textbooks: the Case of Physics?
}

\author{
Fouzia EL-ouazzani ${ }^{1}$, Nadia Benjelloun ${ }^{2}$ \\ ${ }^{I}$ (Interdisciplinary Research Laboratory in Science and Technology Didactics (IRLISTD), Faculty of Science \\ Dhar Mehraz, University Sidi Mohamed Ben Abdellah, Fez. Morocco) \\ ${ }_{2}^{2}$ (Interdisciplinary Research Laboratory in Science and Technology Didactics (IRLISTD), Faculty of Science \\ Dhar Mehraz, University Sidi Mohamed Ben Abdellah, Fez. Morocco)
}

\begin{abstract}
This work focuses on the role and the place given to the history of science and contextual situations in teaching and learning of physics in high school in Morocco. The study is conducted through a quantitative and qualitative analysis of the corresponding physics textbooks. The results show that textbooks give a modest place to contextual situations. We also noticed a very low presence of the history of science in physics textbooks. These results may provide proposals to the various fields of research in physics education, and the improvement of curricula and textbooks for effective science learning.
\end{abstract}

Keywords: History of science, epistemology, didactic, contextual situations, effective science learning.

\section{Introduction}

The National Charter of Education and Training (COSEF, 1999) is the reference framework that reflects the foundations and principles of Moroccan education, which are in turn declined in terms of content According to the specifications, in order to develop textbooks. In 2007, there was an overhaul of textbooks in all subjects, from elementary to baccalaureate, to adapt problem-based learning to the pedagogical adoption of the competency-based approach. This is due to the training needs of the professional community and the social and political demand for new educational models. Create a vibrant school, open to its social, economic and cultural environment, which offers children and young people the opportunity to acquire the values, knowledge and skills that prepare them to integrate into active and professional life

This evolution aims at giving meaning to classroom learning in order to increase interaction with the learner's real environment in order to build an active, virtuous, dynamic and effective citizen who can engage in the prosperity of his country and the whole world.

The teaching of science, and in particular the physical sciences, occupies a special place as a lever for development, hence the need to promote scientific research. In this perspective, the history of science is a rich laboratory in tools and research methods, which can always be inspired. In addition, Bouasse (LOCQUENEUX R., 2005) thinks that a researcher can't economize history if he doesn't want to condemn himself to find what he knew beforehand; also, in his teaching, when he touches on areas that remain open, and in his research, before approaching the study of a phenomenon.

This scientific teaching focuses on the learning of knowledge and the scientific approach. Thus, it is based on the construction of scientific notions that are linked to what is already acquired by the learner in the form of representations and conceptions. We talk about conceptions when we want to list the representations of learners of scientific knowledge in a specific field.

Numerous studies have also revealed that the difficulties encountered by students today are similar to those encountered by scientists in the past. (Azzaoui H. et al, 2015, Guedj M., 2005; Hulin N. 2005; Rosmorduc, 1987; Martinand, 1993). Indeed, these conceptions have been forged over the years either from school situations or from situations of everyday life. This gives resemblances but also divergences because of the differences in teaching and the cultural and technical contexts of our pupils and consequently there is no strict parallel between these difficulties. Some cognitive impediments brought up by didacticians can be compared with the epistemological obstacles that Bachelard (1938) identified in his analysis of the transition from the pre-scientific to the scientific period. This proves the interest of a return to the history of science and to consider it as a didactic tool to overcome these obstacles.

This new system is designed to motivate learning through the activation of previous knowledge and the relevance of context (what context is it in textbooks?).

The history of science can contextualize learning situations by different methods. This makes it necessary to give it a growing place in the scientific curriculum, whether for school or university education or for the training of teachers.

Our work is based on a careful reading of textbooks of physical sciences at the secondary level, which deals with the teaching situations used. In this study, we try to answer the following questions: 
$>$ To what extent have the objectives of reforming the Moroccan education system in relation to contextual situations and those of epistemology and the history of science been achieved by the new physics textbooks?

$>$ What is the place devoted to the history of science in textbooks?

$>$ To what extent did the didactic discourse of textbooks take into account the history of science?

\section{Theoretical Frame}

From ancient times to the present, scientific learning has encountered difficulties and obstacles that limit the understanding of its knowledge and scientific popularization. Several studies have attempted to approach this problematic by highlighting didactics, epistemology and the history of science, which opens the way to a new didactic of the history of science.

Bouasse (LOCQUENEUX R., 2005) states that there are two ways to teach physics, historical method and dogmatic method and that: "The first is to show how ideas were made, how they took a kind of real existence... ". It is the method of a rediscovery of a scientific knowledge, which can be identified by a constructivist approach.

Confessing that it is difficult to expose the whole results on phenomena. But other uses may arise, depending on the case, to make students live a historical controversy by analyzing the nature of the arguments at stake, by presenting the actors, the links between these actors, exchange. This develops various specific and transversal skills among learners, such as communication, persuasion and defense of opinion by arguments ... in addition to the acquisition of a scientific culture.

In this perspective, history has a dual didactic and pedagogical interest, which can be taught or convened to build scientific knowledge. The history of science is an irreplaceable source of reflection on the elaboration of physical concepts and principles, and hence on theories and methods in physics. It teaches us that there is a continuous evolution of physical theories and provides us with examples of scientific approaches whose analysis can contribute to the training of the physicist and the engineer and to the formation of the scientific spirit.

Guiding and directing a learner to build a knowledge based on his own conceptions taken from his real environment, even the false ones, which prevent the understanding and validation of this new knowledge, is a priority objective of socio constructivist theory. This new paradigm attaches great importance to the contextualization of teaching situations, while emphasizing the social aspect. Indeed, the discovery of reality, the search for understanding of natural phenomena and social relations are the driving forces of a scientific activity. Consequently, the social, cultural, economic and political context influences the development of scientific knowledge, which cannot be isolated from the context of knowledge. This is why this theory uses the situation-problem as an instrument.

In order to identify the place given to the history of science and how to use it, and the contextual situations used to introduce scientific concepts and laws into textbooks, we conducted a descriptive analysis. To verify the effectiveness of the history of science and to highlight its use, we have identified the different roles it can have in the teaching of physics (Based on a bibliographic literature).

The importance of these roles varies according to the target school level and the expected learning objectives.

$\checkmark$ R1: Inserting science into history and society and considering it as a "living social construction" by recalling the repercussions of scientific knowledge and models (BACHELARD, G., 1938; De Broglie, L., 1956; PASTEUR, L. 1858).

$\checkmark \quad$ R2: Encourage the teacher to be more tolerant and to relativize the student's mistake and to consider it as evidence of a learning disability (De Hosson, C. 2004).

$\checkmark \quad$ R3: Acquiring a scientific culture through the narration and narrative of scientific stories (Langevin, P., 1933; PASTEUR, L. 1858).

$\checkmark$ R4: Develop communication and argumentative skills by creating a scientific debate (controversies) (BACHELARD, G., 1938; BRUNOLD CH. 1958).

$\checkmark \quad$ R5: Locate events with historical context and identify scholars to students (GUEDJ, M., LAUBE, S., \& SAVATON, P., 1957).

$\checkmark \quad$ R6: To give meaning and taste to learning and to stimulate students' motivation by telling stories about the concepts, theories and laws studied (Mayrargue, A. \& Savaton, P. 2006).

$\checkmark \quad$ R7: Identify epistemological barriers in history and class practices. (GIVRY, D., 2003 ; BACHELARD, G., 1938).

$\checkmark \quad$ R8: Draw inspiration from how obstacles were overcome to overcome those faced by students (Laugier, A. \& Dumon, A. 2001; BACHELARD, G. 1938).

$\checkmark$ R9: Building scientific knowledge by putting the student in a problem situation or using historical controversy or replication of historical experience (MACHEREY, P. 2007; De HOSSON, C. 2004). 
$\checkmark \quad$ R10: Teaching the scientific process by considering the student as a researcher who analyzes the scientist's approach in his work or replicates a historical experience (Halbwachs, F. 1974).

$\checkmark \quad$ R11: Promote scientific research and popularization of science. (LOCQUENEUX, R. 2005).

$\checkmark$ R12: To train the scientific and critical minds of learners (BACHELARD, G., 1938; Halbwachs, F., 1974).

\section{Methodology}

The textbook in Morocco is an educational tool for pupils, but also designed for teachers and chosen by them. However, I note (An ongoing study) the reluctance of students to use it. By arguing that they have difficulties to understand the activities proposed individually, since their teachers do not use it in the classroom. This means of education remains unfeasible and ineffective in the teaching-learning process.

This gap between textbooks and their users can turn into eternal rupture with books in general. This has serious consequences on the scientific, cultural, communicative and social training of a person and therefore on citizenship. The textbooks which we are going to analyze have been used for our teaching in the physical sciences. In order to try to approach this problem and to take account of the place given to the history of science (especially physics) in textbooks, since it is certain that the history of science contributes and can develop these aspects in the pupils (BRUNOLD CH. 1958; Mayrargue, A. \& Savaton, P., 2006).

We conducted a thorough re-reading in order to obtain a relevant analysis.

We have chosen as an object of study the textbooks of physical sciences of college and high school which have for title:

College

$>$ 1st year: «Physical sciences» (BOUDHAR, A., \& al, 2007).

$>$ 2nd year: «Al waha en physique» (MOUTAKILLAH, M., \& al, 2007).

$>$ 3rd year: «Al massar en physique» (BENSADIK, A., \& al, 2007).

High school

$>$ The common scientific core «Mourchidi in physics and chemistry», «Al Massar in physics» (BENSADIK, A., \& al, 2007; MARZOUK, \& al, 2007).

$>1$ st year of the baccalaureate «Almassar en physique», «Al fadaa en physique» (BENSADIK, A., \& al, 2007; BOUAYAD, A., \& al, 2007).

$>$ 2nd year of the baccalaureate «Almassar en physique», «Al fadaa en physique» (BENSADIK, A., \& al, 2007; BOUAYAD, A., \& al, 2007).

\section{Instrumentations}

The analysis of textbooks is descriptive; it includes information related to disciplinary fields and their modes of introduction. It contains information on the nature and contextualization of learning activities and exercises. We have determined the degree of presence of the history of the physical sciences for each textbook and for each field (mechanics, electricity, electrodynamics, optics, wave and nuclear) in terms of historical situation. We have focused on the problem situations proposed in textbooks. For the qualitative analysis of the content of these historical situations, we have noted the different roles accorded to the history of science used to measure its wealth, utility and quality.

\section{Result}

The first reading shows that before starting each part, the learner is invited to discover: the skills and objectives envisaged the plan, the extensions and interactions with other subjects and a reminder of his prerequisites. Thus most of these manuals use, in order to introduce each part and each unit, a "situationproblem", sometimes it is used for a paragraph of a lesson.

Each unit is a set of documentary and practical activities to be carried out in class, followed by a synthesis of laws and concepts and constructed knowledge. At the end a series of varied exercises (exercises of application and problems) is proposed.

These activities aim to involve the learner in a motivating situation that excites, activates and stimulates his / her prerequisites or poses a problem situation in order to build a new knowledge. The purpose of the exercises is to acquire a knowledge or know-how from the learner, or to check whether this knowledge or know-how has been acquired (What about the values to be transmitted).

Although official instructions emphasize the contextualization of learning situations and the increased interaction with students' physical and social environments, the emphasis on contextual situations in textbooks is other situations.

For the proposed problem situation, most textbooks use an image with commentary followed by questions. 
This brief analysis addresses the validity and effectiveness of the situation-problem function proposed to students in textbooks.

The second reading aims to identify the place of the history of physics in textbooks and its use to build scientific knowledge.

\section{College Level:}

College students are invited to discover and study four parts of physics / chemistry, titled as follows: Matter and the environment, electricity, light and image, and mechanics.

An analysis of college textbooks allows us to distinguish two types of textbooks:

- The textbooks of the 1st and 2nd years (BOUDHAR, A., \& al, 2007; MOUTAKILLAH, M., \& al, 2007) give no place to the history of science.

- The 3rd year textbook (BENSADIK, A., \& al, 2007) gives a small place to the story just to make a historical reminder of some events and present some scientists. Indeed, to describe the constituents of the atom in chronological order, beginning with the idea of Democritus "matter is made up of indivisible particles ..." until the discovery of the positive charges of the nucleus. The same approach to introducing the discovery of universal interactions by citing the works of Nicolas Copernicus, Nico Brahi, Keppler and Isaac Newton. Additions like "the history of science: I know a scientist" refer to the portrait of the "scientist" (Jams Watt, Jams Joule), whose dates of birth and death are indicated. These additions are intended to situate events and to know "scholars", without historical context. This type of approach is intended, as it were, to awaken curiosity and inform.

\section{High school level:}

High school students are invited to deepen their knowledge of physics also to apply and fix the experimental scientific method.

\subsection{Common core scientific}

The students of the common core begin their studies by mechanic; a modest place is reserved for history. Indeed, a text tells the story related to the discovery of the forces of universal gravitation by Newton which explains the movement of the stars around the sun and the moon around the stars, is accompanied by a photo of Isaac Newton.

Similarly, to approach the concept of relativity of movement a truncated text is used, which has the same principle of Einshtein's statement, to create a debate between the students on the movement of the train with respect to the observers. This method is suitable for building knowledge by the students themselves.

For the part of electricity, no history is mentioned just purely practical activities using software.

\subsection{1st year of the baccalaureate}

The students of 1st year continue to deepen their knowledge in mechanics by approaching the 1st part of the mechanical work and energy. A tube experiment of Newton is proposed, to study the free fall of bodies in a vacuum. It is a way to redo a historical experience.

In the second part of Electrodynamics, a historical overview of magnetism from Aristotle to Shen Guai, in addition to a historical textural detailing of the magnetic field induced by a charged wire.

Thus practical activities of a historical nature consist in repeating experiments by the learners, like Hans Kristian Oersted's experience and that of Descartes in order to discover the lines of the field formed by the dust of the iron powder. This type of work consists of a rediscovery of a scientific knowledge; the learners themselves build their knowledge. The history of science has a double interest: pedagogical and didactic.

For the third part of the optics two historical insights are proposed. The first summarizes the progression of the astronomical telescope with the Arab scholar Ibn el Haytame and Gallilé and the second quotes the events related to the progression of the telescope with James Grégory, Isaac Newton and Foucault. As already mentioned, these texts serve to awaken students' curiosity, to inform and to acquire a scientific culture.

\subsection{2nd year of the baccalaureate}

The questions and problems dealt with by physics are neither studied nor discussed till the 2nd year of the baccalaureate, such as an introduction entitled "Introduction to physics: questions posed to physicists". Documentary activities are made available to the students to make them recognize the physics and its foundations by the statements of Alain Aspect et al (2004) and Stephan Hawking (2005). These two physicists are presented by their dated photos. Similarly, history is called just to get a scientific culture as the case of a literary culture. 
No history is associated with the first part of the waves. On the other hand, in the second part of the nuclear transformations, it is a question of telling two short stories: one of the discovery of radioactivity by Henri Becquel and the other of the discovery of the nuclear rays whose heroes are Marie and Pierre Curie and Paul Villard.

A "reading" paragraph deals with natural radioactivity and Radon 222 is proposed. This is another way to encourage pupils to read and communicate the scientific language. This same type of "reading" paragraph is intended in the part of electricity (electricity in the atmosphere) to revive the experience of Benyamine Frankeline.

These results show that the rate of teaching situations (activities and exercises) using history in textbooks, is small and limited to activities only. This clearly indicates that teaching which takes account of epistemology and the history of science occupies very little place in the textbooks. We also note that the distribution of historical situations is uneven across disciplinary fields. It is clear that activities using the history of science are more present in the nuclear field than in other fields, on the other hand, we notice their total absences for the waves.

Moreover, qualitative analysis allows us to conclude that the dominant roles given to the history of science are to acquire a scientific culture and to situate events but without a historical context, also to identify scientists to the students, followed by the role of developing communication and argument skills. The means used by the designers of these textbooks, to narrate an event, are: a place, a man, a date, a photograph, without a historical context. This allows us to deduce that these designers hide a misunderstanding of their subject and its history. The introduction of the history of science into these books is therefore concerned only with the success of research and discovery. Unfortunately, we have noticed a total absence of historical activities that treat the conceptual difficulties encountered at a given time. This could have enabled us to identify the epistemological obstacles and to demonstrate the similarities between the difficulties encountered by scientists in the past and those encountered by learners today. In addition, history is rarely used as a tool to build scientific knowledge or to teach the scientific process.

\section{Discussion}

New curricula and textbooks promote teaching by problem, which is based on constructivist principles, and is aimed at learning from a problem-based point of departure. On the other hand, program designers simply list a "skills" without focusing on "problem situations". While the textbook designers illustrated the problem situation with an image accompanied by a commentary followed by the questions to be answered, leaving this important work to the teachers. Nowadays, the problem is that it is the only way to get the best out of the knowledge and experience of the students. It is only after identifying the obstacle (s) that we are looking for the most appropriate problem-situation and not the intended competence, and we are talking about problems and problems. Without going into details, the application of the competence approach in Morocco. This analysis allows us to conclude that the contextual situations (situation-problem) do not have the deserved place in the recasting of these manuals nor the realization of the expected objectives.

This instrument not only serves to construct new knowledge and achieve the teaching objective, but also to teach the experimental approach and to form the scientific spirit. (Pupil-student-teacher), to avoid vertical transmission, from teacher to pupil, scientific knowledge, just to advance in the presentation of the content prescribed in the program. In this perspective, the objectives of using problem situations and the history of science are compatible and integrated. This can translate into historical situations-problems.

In this context, one can draw inspiration from the history of science to create problem situations, considering it as a generating and rich source or rather a "database of problems and problems".

On the other hand, the dominance of the mathematical character in relation to the physical character causes learning difficulties in the learners in the conceptions and phenomena studied. The history of science can be seen as a tool to stimulate students' motivation and promote retention and understanding of physical phenomena (a tool for students). Moreover, the history of science allows us to extract the difficulties encountered by scientists in the past, and thus to deduce those that can meet the learners today. This helps the teacher construct situations that allow learners to overcome the obstacles encountered by them, drawing inspiration from the way obstacles have been overcome (a tool for the teacher). In this sense, we consider that its weak presence in textbooks will have a negative impact on the construction of scientific knowledge among learners. Thus, the results showed that history has not fulfilled all the roles intended for it, and that its use is not equal to the great objectives expected in the construction and acquisition of scientific knowledge.

In analyzing these manuals, we found that they guide students to use new information and communication technologies (ICTs). This technology provides innovative ways to explore learning strategies and promotes a pedagogical approach that puts the student at the center of the teaching-learning process. In this context, we consider that ICT can enrich teaching situations based on the history of science. As a non-limiting example, MOOCs or reverse classes can be created, simulations can be made to develop learning sequences 
based on the history of science to benefit from the advantages of both without losing school time. This requires the mastery of the use of ICT by teachers.

Finally, on the basis of the results of this analysis, we can consider practical proposals:

i. Adjust physics curricula and textbooks on the introduction and exploitation of historical and contextual teaching situations.

ii. Provide more comprehensive and relevant initial training on strategies and teaching methods based on the history of science to facilitate the work of teachers within the classroom.

iii. Strengthen teachers' knowledge of the history and epistemology of science through complementary continuing education.

iv. Train teachers on the use of information and communication technologies and provide the necessary digital resources and materials, facilitating the adoption of a didactic of the history of science through ICT.

v. Organize continuous training and formative meetings and events to present the dual pedagogical and didactic interest of the history of science for the implementation of this approach.

vi. Create a computerized database or database to facilitate access to different historical documents and teaching situations.

\section{Conclusion}

The results of the analysis of physical science textbooks in Morocco show that the history of science today occupies a modest place. Despite the different methods of implementing historical elements, the use of history does not, in most cases, go beyond the narration of historical events and discovery stories, 'Scientific culture'. Rarely, it is called to build and acquire scientific knowledge. We also found a low contribution of problem situations. Moreover, the chosen context does not address the resolutions of the real problems of the learner's environment. On the other hand, the history of science makes it possible to treat the problems that humanity has been confronted, so to understand the why of certain practices, to discover simple and robust methods, which can still be inspired today to solve many problems.

By using ICT to introduce the history of science in physical science learning, the benefits of both approaches can be realized for effective and non-traditional learning, based on the history without wasting time.

Our results serve as a basis for reflection on how to use the history of science to acquire new scientific knowledge and on the construction of teaching situations, taking into account the contextual, epistemological and historical nature of scientific knowledge.

\section{References}

[1] Special Committee of Education and Training (SCOET). (1999). National Charter for Education and Training. Rabat: MEN. Available online at: http: // uh2c.ac.ma/uh2c/loi/charte_en.pdf

[2] LOCQUENEUX, R. (2005). The interest of the history of physics for the training of physicists according to Henri Bouasse. In: Revue d'histoire des sciences, tome 58, $\mathrm{n}^{\circ} 2$ 2, 2005. pp. 407-431.

[3] AZZAOUI, H., BENJELLOUN, N., and al. (2006). Analysis of the conceptions of senior secondary school physics teachers in Morocco, about the inertial motion as a motion-state, IOSR Journal of Research \& Method in Education, Volume5, Issue 4, pp 4348 http://www.iosrjournals.org/iosr-jrme/pages/v5-i4.ve.html.

[4] GUEDJ, M. (2005). Use historical texts in the teaching of physical sciences in the second grade of French secondary school. Didaskalia, 26, 75-95.

[5] HULIN, N. (2005). History of science and science education in the Secondary School of the Third Republic / History of science and science teaching in secondary schools under the Third Republic. In: Revue d'histoire des sciences, tome 58, ${ }^{\circ} 2$, 2005. pp. 389405.

[6] ROSMORDUC, J. (1987). Can the history of physics illuminate epistemological obstacles? Aster, 1987_5_117ob.

[7] MARTINAND, J. (1993). History and didactics of physics and chemistry: what relations, Didaskalia, $\mathrm{n}^{\circ} 2-1993$.

[8] BACHELARD, G. (1938). The formation of the scientific mind. Paris, Vrin.

[9] De Broglie, L. (1956). On the Paths of Science, Paris, 1956, p. 377.

[10] PASTEUR, L. (1858). Works of Pastor. Tome 7, Paris 1939, p.

[11] De Hosson, C. (2004). Contribution to the analysis of the interactions between history and didactics of sciences. Development of a teaching medium for the optical vision mechanism for the primary school and the college and the first elements of evaluation.

[12] Langevin, P. (1933). Thought and Action, Editions Sociales, p.192.

[13] BRUNOLD, CH. (1958). Role of History in the teaching of Physical Sciences. In: Review of the history of sciences and their applications, volume $11, \mathrm{n}^{\circ} 2,1958$. pp. 97-107.

[14] GUEDJ, M., LAUBE, S., \& SAVATON, P. (1957). Elements of problematic and methodology for a didactics of epistemology and the history of sciences and techniques (EHST). Journal of the History of Science, (X, 1957, No. 3, pp. 193-196)

[15] Mayrargue, A. \& Savaton, P. (2006). "What are the links between the history of science, epistemology and the didactics of disciplines? », Tréma [Online], 26 | 2006. URL: http://trema.revues.org/98; DOI: 10.4000 / trema.98

[16] GIVRY, D. (2003). The concept of mass in physics: some ideas about conceptions and obstacles. Didaskalia, No. 22. 2003. pp. 4167

[17] Laugier, A. and Dumon, A. (2001). In search of epistemological obstacles to the construction of the concept of chemical element by students of second. Didaskalia, Paris, 2001, no. 22, pp. 69-97.

[18] MACHEREY, P. (2007). History of knowledge and epistemology. Review of the history of science. Tome 60-1 2007. pp. 217-236

[19] De Hosson, C. (2004). Historical controversy: a didactic tool "Part 1: Dialogue on the ways in which vision is made". BUP, No. 866, pp. 1057-1068.

[20] Halbwachs, F. (1974). Physical Thinking in Children and the Scholar, ed. Delachaux and Niestlé. 
[21] BOUDHAR, M., BROUK, A., \& AMLAHOUCH, M. Physical sciences. Preparatory year (Manual in Arabic). (2007). Morocco: Publishing and distribution company Al Madariss.

[22] MOUTAKILlAH, M., BOUBKR, A., \& WIJER, M. Al Waha, Physics. 2nd preparatory year (Manual in Arabic). (2007). Morocco: Publishing and distribution company Al Madariss.

[23] BENSADIK, A., LMSSAWAB, M., \& CHOUIDRA, A. (2007). Al massar, physics, 3rd preparatory year (Manual in Arabic). Morocco: Printing Nadia Edition Rabat.

[24] BENSADIK, A., LMSSAWAB, M., \& CHOUIDRA, A. (2007). Al massar, physics, common core (Manuel in Arabic). Morocco: Printing Nadia Edition Rabat.

[25] MARZOUK, et al. (2007). Mourchidi in Physics and Chemistry (Manuel in Arabic). Morocco: Printing.

[26] BENSADIK, A., LMSSAWAB, M. \& CHOUIDRA, A. (2007). Al Massar, Physics, 1st year BAC (Manuel in Arabic). Morocco: Printing Nadia Edition Rabat.

[27] BOUAYAD, A., et al. (2007). Al fadaa, physics, 1st year BAC (Manuel in Arabic). Morocco: Impression El Maarif Al Jadida Rabat.

[28] BENSADIK, A., LMSSAWAB, M., \& CHOUIDRA, A. (2007). Al massar, physics, 2nd year BAC (Manuel in Arabic). Morocco: Printing Nadia Edition Rabat.

[29] BOUAYAD, A., et al. (2007). Al fadaa, physics, 2nd year BAC (Manuel in Arabic). Morocco: Impression El Maarif Al Jadida Rabat.

[30] ASPECT, A., BALIAN R., et al. (2004). Tomorrow, physics. Odile Jacob 2004.

[31] HAWKING, S. (2005). A beautiful story of time. Flammarion, 2005. 